\title{
Biophysical Model for Beat-to-Beat Variations of Vectorcardiogram
}

\author{
E. A. I. Aidu, V. G. Trunov, L. I. Titomir \\ Institute for Information Transmission Problems, Russian Academy of Sciences, \\ Bolshoy Karetny per. 19, Moscow, 127994, Russia, aidu@iitp.ru
}

\begin{abstract}
Linear deformation of the myocardium results in a linear transformation of orthogonal electrocardiosignals under the condition of topological invariability of the depolarization and repolarization processes in the myocardium. The relation between the linear transformations in the physical and functional spaces was found. Several practically important conclusions follow as a logical consequence of these statements. The parameters of linear transformations of the vectorcardiogram (eigenvectors, eigenvalues, rotation angles, determinants) may be used as indices of the heart position and haemodynamic changes.
\end{abstract}

Keywords: vectorcardiogram, biophysical model, beat-to-beat alteration

\section{INTRODUCTION}

Con OMPARISON OF single beats within one electro cardiographic (ECG) recording or consecutive ECG recordings from the same patient during cardiologic evaluation and in long term monitoring is an essential part of the contemporary non-invasive electrocardiologic diagnosis.

Variations of the ECG signals from one heartbeat to another are affected by a number of factors, which do not reflect the electrophysiological state of myocardium itself and complicate the analysis of ECG signals. These factors are usually treated as distortion or extracardiac factors [1]. Considering the comparison of consecutive ECG cycles from the same patient, the main distortion factors are the patient's breathing movements, possible changes in position, and haemodynamic alterations invoking (arousing) rotation and deformation of the myocardium.

A simple biophysical model of excitable media is proposed to describe the dipole moment changes after deformation of the excitable media (myocardium). The first approximation of the deformation is linear transformation.

\section{SUBJECT \& MODEL}

Suppose that the excitable medium (region M, Fig.1) was deformed by the linear transformation $\mathbf{T}$ (region $\mathbf{M}_{\mathrm{T}}$, Fig.1):

$$
\rho=\mathbf{T} r
$$

where $\rho=(\xi, \eta, \zeta)^{\prime} \in \mathrm{M}_{\mathrm{T}}$ is the new position of the myocardium point $r=(x, y, z)^{\prime} \in \mathrm{M} \quad$ after transformation $\mathbf{T}$ ( ', transpose symbol). Let $d, \mathrm{~J}(r)$ and $d_{\mathrm{T}}, \mathrm{J}_{\mathrm{T}}(\rho)$ be the dipole moments and current densities before and after the transformation $\mathbf{T}$, respectively. The dipole moment vectors are expressed as integrals of current density over the excitable media regions:

$$
d=\int_{\mathrm{M}} \mathrm{J}(r) \mathrm{d} v_{r}, d_{\mathrm{T}}=\int_{\mathrm{M}_{\mathrm{T}}} \mathrm{J}_{\mathrm{T}}(\rho) \mathrm{d} v_{\rho} .
$$

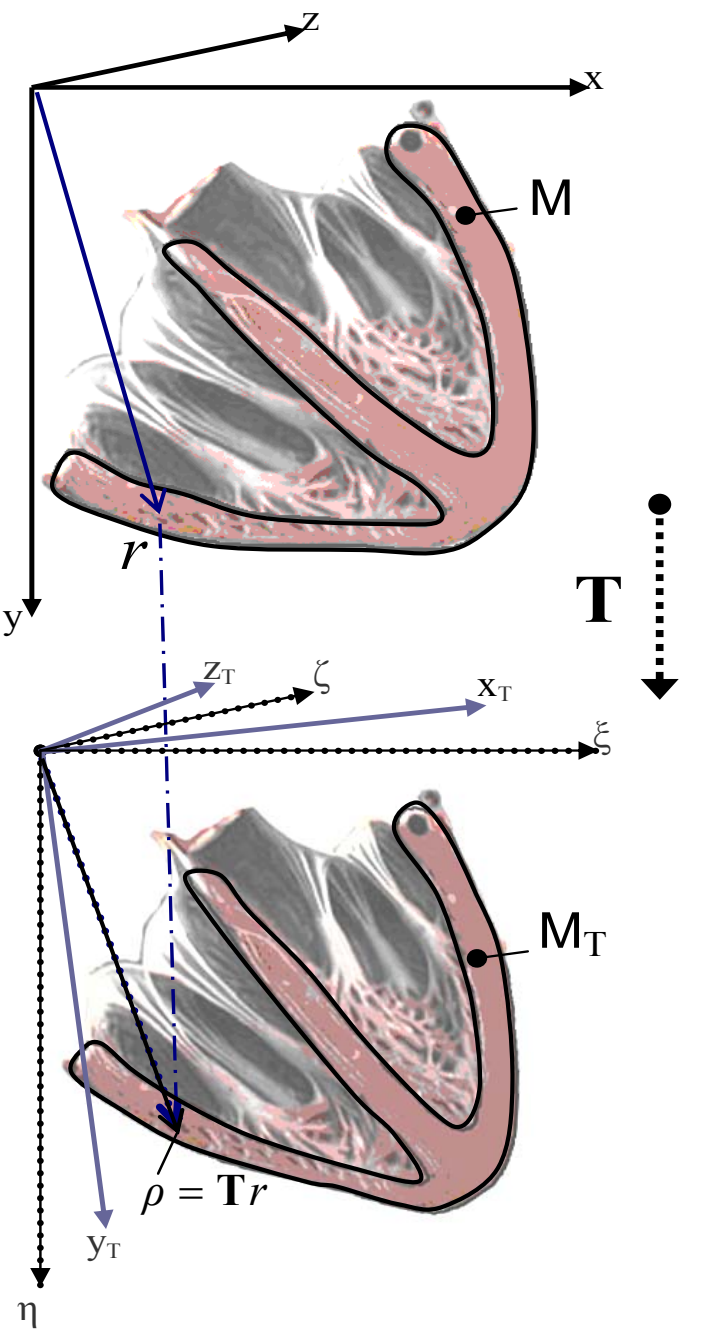

Fig.1 Deformation of myocardium. The point $\rho=(\xi, \eta, \zeta)^{\prime} \in \mathrm{M}_{\mathrm{T}}$ is the new position of the myocardium point $r=(x, y, z)^{\prime} \in \mathrm{M}$ after transformation $\mathbf{T}$. 
For the bidomain model of the excitable medium the current density is determined by gradient of the transmembrane potential [2], then before and after transformation $\mathbf{T}$ the current densities are:

$$
\mathrm{J}(r)=-\sigma_{\mathrm{i}} \nabla_{r} \mathrm{U}(r), \mathrm{J}_{\mathrm{T}}(\rho)=-\sigma_{\mathrm{i}} \nabla_{\rho} \mathrm{U}_{\mathrm{T}}(\rho),
$$

where $\sigma_{\mathrm{i}}$ is the intracellular conductivity; $\mathrm{U}(r), \mathrm{U}_{\mathrm{T}}(\rho)$ are the transmembrane potentials before and after transformation $\mathbf{T} ; \nabla_{r}=\left(\frac{\partial}{\partial x}, \frac{\partial}{\partial y}, \frac{\partial}{\partial z}\right) ; \quad \nabla_{\rho}=\left(\frac{\partial}{\partial \xi}, \frac{\partial}{\partial \eta}, \frac{\partial}{\partial \zeta}\right)$.

Assume further that the state of myocardial cells does not change after transformation $\mathbf{T}$, and transmembrane potential remains the same, so

$$
\mathrm{U}_{\mathrm{T}}(\rho)=\mathrm{U}_{\mathrm{T}}(\mathbf{T} r)=\mathrm{U}(r) .
$$

The main aim of the present reasoning is to obtain the relation between dipole moments before and after the deformation of the excitable medium. Using (1) - (4), taking into account that gradient of a function is a contravariant tensor of rank 1, after several simple manipulations and change of variables $r=\mathbf{T}^{-1} \rho$, the relation is obtained:

$$
\begin{aligned}
d_{\mathrm{T}} & =\int_{\mathrm{M}_{\mathrm{T}}} \mathrm{J}_{\mathrm{T}}(\rho) \mathrm{d} v_{\rho}=-\sigma_{\mathrm{i}} \int_{\mathrm{M}_{\mathrm{T}}} \nabla_{\rho} \mathrm{U}_{\mathrm{T}}(\rho) \mathrm{d} v_{\rho}= \\
& =-\sigma_{\mathrm{i}} \int_{\mathrm{M}}\left(\mathbf{T}^{\prime}\right)^{-1} \nabla_{r} \mathrm{U}(r) \frac{\partial v_{\rho}}{\partial v_{r}} \mathrm{~d} v_{r}= \\
& =-\sigma_{\mathrm{i}}\left(\mathbf{T}^{\prime}\right)^{-1}|\mathbf{T}| \int_{\mathbf{M}} \nabla_{r} \mathrm{U}(r) \mathrm{d} v_{r}= \\
& =\left(\mathbf{T}^{\prime}\right)^{-1}|\mathbf{T}| d, \quad|\mathbf{T}|=\operatorname{det}(\mathbf{T}) .
\end{aligned}
$$

\section{RESUltS \& COROLLARIES}

For the bidomain model a linear deformation $\mathbf{T}$ of the excitable media leads to the linear transformation $\mathbf{G}$ of the dipole moment under the condition of topological invariability of the activation propagation. The relation between these two linear transformations is:

$$
d_{\mathrm{T}}=\mathbf{G} d, \quad \mathbf{G}=\left(\mathbf{T}^{\prime}\right)^{-1}|\mathbf{T}| .
$$

Assume further that the vectorcardiogram (VCG) reflects the heart vector evolution during the depolarization process in the heart. Let $\left\{\mathbf{f}_{0}(t), \mathbf{f}_{1}(t), \ldots, \mathbf{f}_{n}(t)\right\}$ be a sequence of $\mathrm{n}$ QRS loops (Fig.2A), and $\mathbf{G}_{i}$ be the linear transformation that maps $\mathbf{f}_{0}$ (reference loop) to approximate $\mathbf{f}_{i}$ with the minimum root mean square error $\varepsilon_{i}$ :

$$
\mathbf{f}_{i}=\mathbf{G}_{i} \mathbf{f}_{0}+\mathbf{e}_{i}, \quad \varepsilon_{i}=\left\|\mathbf{e}_{i}\right\|, i=1, \ldots, n .
$$

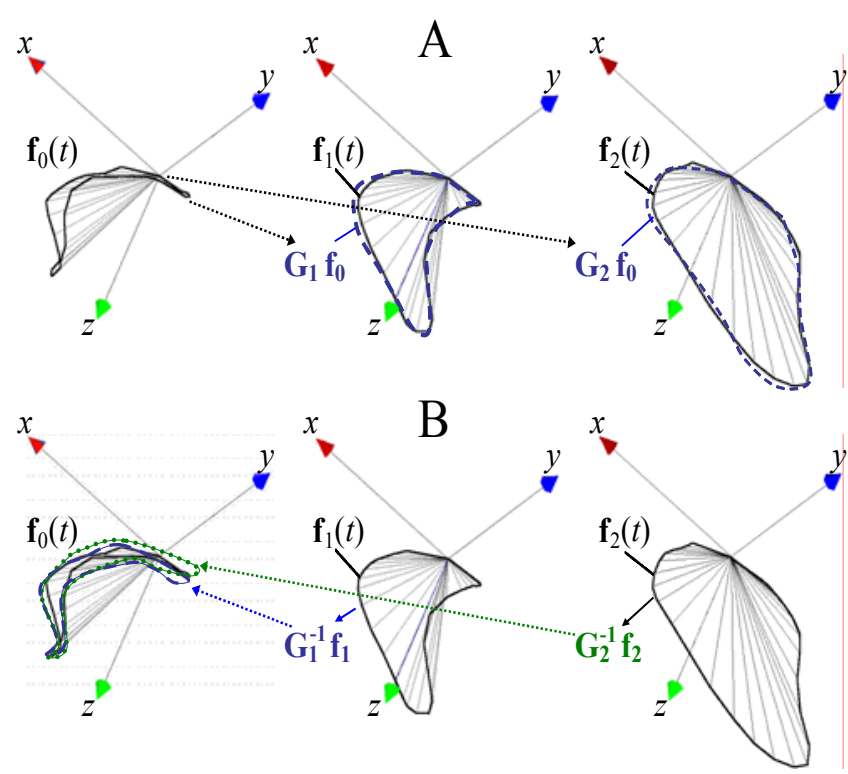

Fig.2 Approximation of the QRS loops by linear transformations of the reference loop (A), and normalization of the QRS loops to eliminate extracardiac factors (B).

The inverse transformations $\mathbf{G}_{i}^{-1}$ applied to the corresponding QRS loops give curves that are similar to the reference loop and lie quite near it (Fig.2B).

The transformations $\mathbf{G}_{i}$ of the reference QRS loop are represented by the rotations $\mathbf{O}_{i}$ and dilatations $\mathbf{S}_{i}: \mathbf{G}_{i}=\mathbf{O}_{i} \mathbf{S}_{i}$. Any linear transformation in the Euclidean space may be obtained by sequential executing rotation and dilatation (in any sequence). This provides a separate analysis of these two kinds of transformations. The rotation of the reference QRS loop is characterized by the eigenvector of this transformation and the angle of turn around this vector.

The dilatation of the reference QRS loop is characterized by the coefficients of dilatation along three orthogonal directions, or, in other words, by the eigenvalues $\left(\sigma_{i 1}, \sigma_{i 2}, \sigma_{i 3}\right)$ and three orthogonal eigenvectors of this transformation. The product of these three eigenvalues is equal to the factor of the volume expansion for any threedimensional body after the transformation.

Looking back to the corresponding myocardium transformations $\mathbf{T}_{i}$ through (6), it is possible to calculate the eigenvalues $\left(\lambda_{i 1}, \lambda_{i 2}, \lambda_{i 3}\right)$ and eigenvectors that characterize the myocardium expansion (or contraction) for the $i$-th beat against reference beat. The product of eigenvalues $\lambda_{i}=\left|\mathbf{T}_{i}\right|=\lambda_{i 1} \lambda_{i 2} \lambda_{i 3}$ may be treated as the end-diastolic heart volume dilatation (or contraction) relative to the reference beat.

The simple relations between the eigenvalues of linear transformations in the physical and functional spaces are obtained using (6): 


$$
\begin{gathered}
\sigma_{i 1}=\lambda_{i 2} \lambda_{i 3}, \sigma_{i 2}=\lambda_{i 1} \lambda_{i 3}, \sigma_{i 3}=\lambda_{i 1} \lambda_{i 2} \\
\sigma_{i}=\lambda_{i}^{2} \text { or }\left|\mathbf{G}_{i}\right|=\left|\mathbf{T}_{i}\right|^{2}
\end{gathered}
$$

The same results may be obtained when analyzing the double layer activation front deformation (Fig.3), and for more general cases. The dipole moment is proportional to the integral of the transmembrane potential over the closed surface of the excitable medium region. Calculation of the relation between dipole moments before and after transformation and integration over the whole surface of excitation gives the same equations (6), (8).

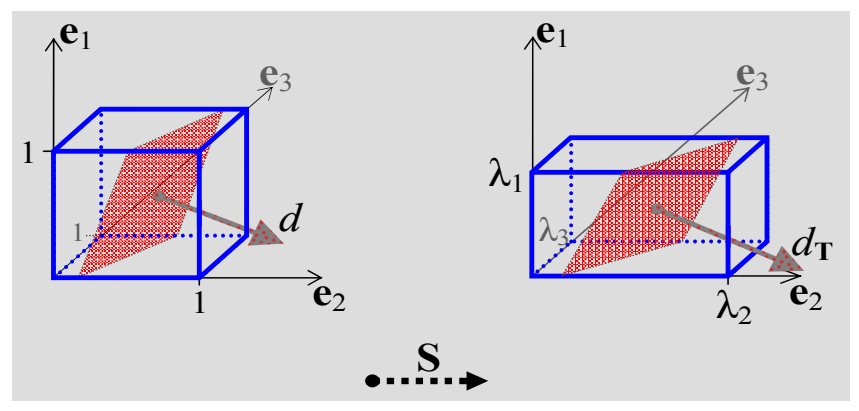

Fig.3 Elementary cube and surface element dilatation along three eigenvectors of transformation $\mathrm{S}$.

\section{DISCUSSION}

The influence of the ventricular volume on the electric heart vector is usually explained by the Brody effect [1], not mentioning the changes of myocardium that contain the sources of the electric field. The Brody effect means that the intracardiac blood surface plays the role of an imperfectly reflecting curved mirror. Changes in VCG are caused both by myocardial deformation and by its reflection on intracardiac blood. It is important to find out their relative input. The same changes of VCG after changes of the left ventricular enddiastolic volume may be easily explained by the Brody effect [1], as well as by the linear deformation of myocardium.

There were at least two experimental investigations that substantiate the proposed model.

In $[3,4]$ the linear transformation along with its polar decomposition into rotation and dilatation and corresponding eigenvectors and eigenvalues were used to describe the left ventricular dynamic geometry in the intact and open chest dogs. This description was in good agreement with experimental measurements, and parameters of its decomposition into dilatation and rotation were physiologically and anatomically meaningful.

The presented model was used in [5], where a set of vectorcardiograms recorded during the parabolic flights of a laboratory aircraft was analyzed. In all normal cases, the QRS loop for each person, as a curve in the three dimensional (3D) vectorcardiographic space, remains unchanged (invariant) after the proper 3D linear transformation (the relative error of approximation was less than 0.05). The distance between the superposed QRS loops served as an indicator of changes in the heart depolarization process. Changes of the QRS volume factor $\left|\mathbf{G}_{i}\right|$ were in accordance with haemodynamic changes due to gravitation acceleration.

\section{CONCLUSIONS}

A simple biophysical model is proposed to describe the dipole moment changes after deformation of the excitable medium (myocardium). Using the bidomain model, it is stated that linear deformation of the excitable medium leads to linear transformation of the dipole moment under the condition of topological invariability of the repolarization and depolarization processes.

The proposed model was experimentally substantiated by measurement in the intact and open chest dogs [3, 4] and by analyzing vectorcardiograms recorded during the parabolic flights of a laboratory aircraft [5].

The parameters of linear VCG transformations (eigenvectors, eigenvalues, rotation angles, determinants) may be used as indices of the heart position and haemodynamic changes.

The real interrelation of VCG transformations and myocardium deformations is complicated. The accuracy of the presented biophysical model and its diagnostical possibilities should be determined in the future theoretical and experimental investigations.

\section{ACKNOWLEDGMENT}

This work was supported by the Presidium of the Russian Academy of Sciences, program "Basic Sciences for Medicine".

\section{REFERENCES}

[1] Malmivuo, J., Plonsey, R. (1995). Bioelectromagnetism: Principles and Application of Bioelectric and Biomagnetic Fields. New York, USA: Oxford University Press.

[2] Miller, W.T., Geselovitz, D.B. (1978). Simulation studies of the electrocardiogram. I. The normal heart. Circ. Res., 43, 301-315.

[3] Walley, K.R., Grover, M., Raff, G.L., Benge, J.W., Hannaford, B., Glantz, S.A. (1982). Left ventricular dynamic geometry in the intact and open chest dog. Circ. Res., 50, 573-589.

[4] Grover, M., Glantz, S.A. (1983). Endocardial pacing site affects left ventricular end-diastolic volume and performance in the intact anesthetized dog. Circ. Res., 53, $72-85$.

[5] Aidu, E.A.I., Trunov, V.G., Titomir, L.I., Capderou, A., Vaïda, P. (2003). Transformation of vectorcardiogram due to gravitation alteration. Meas. Sci. Rev. 3 (2), 2932 . 